\title{
ORIGINAL ARTICLE \\ Development of heterotopic ossifications, blood markers and outcome after radiation therapy in spinal cord injured patients
}

\author{
H Krauss, D Maier, V Bühren and F Högel
}

Study Design: Retrospective study.

Objectives: This study was implemented to detect risk factors for the developing of heterotopic ossifications (HOs) in spinal cord injury (SCl) patients.

Setting: This study was conducted in Murnau, Germany.

Methods: All patients from 2008-2012 with acute SCI were routinely examined by ultrasound of the hips every 2 weeks. The sub group of $\mathrm{SCl}$ patients suffering of $\mathrm{HO}$ of the hips were extracted and the incidence of developing an $\mathrm{HO}$ was calculated. Parameters like age, level of injury, ASIA Impairment Scale (AIS), duration time of accident until diagnosis of HO, Brooker stage, localization of $\mathrm{HO}$ (magnetic resonance imaging (MRI)) and symptoms like thrombosis, emboli, decrease of range of motion (ROM), dermal symptoms, swelling, increase in D-Dimere level, were evaluated. Also accompanying injuries of the brain, lung and extremities were recorded.

Results: From January 2008 until January 2012, 575 patients with an acute and traumatic SCl were treated in our Department. During this period $32 \mathrm{HOs}$ were detected in the muscles surrounding the hip. In 10 cases a single side and in 22 cases both sides were affected. A total of 26 patients were detected showing up a Brooker 0, two patients Brooker 1, and five patients a Brooker stage $>2$. The adductor muscles showed an edema in 19 cases and the quadriceps muscles were affected in 15 cases. $26 \%$ of all SCl patients showed AIS A status, but in patients who developed HO, $64 \%$ have had an AIS A status. $19 \%$ of patients with a HO were AIS B and 9.5\% showed an AIS C and D. Regarding the level of injury the distribution of patients suffering of $\mathrm{HO}$ was comparable to the distribution of $\mathrm{SCl}$ patients without $\mathrm{HO}$. In mean $\mathrm{HO}$ were detected 9 weeks after $\mathrm{SCl}$ and no new $\mathrm{HO}$ were found after the 22nd $(n=1)$ week of injury. Clinical symptoms such as swelling, pain, redness or decrease in ROM or increase in D-Dimere levels were seen in 24 cases. Accompanying injuries like brain injury and lung contusions were found in $83 \%$ of patients developing $\mathrm{HO}$. The incidence of thrombosis was comparable to $\mathrm{SCl}$ patients without $\mathrm{HO}$. One patient with no accompanying injuries or clinical symptoms was detected by routinely performed ultrasound.

Conclusions: The risk of developing $\mathrm{HO}$ in patients with traumatic $\mathrm{SCl}$ is $5.5 \%$ but increases when accompanying injuries of the brain and lung occur. Patients with a neurological status of AIS A must also be quoted as risk patients. When considering the described risk factors and clinical symptoms, $96 \%$ of all $\mathrm{HO}$ can be detected.

Spinal Cord (2015) 53, 345-348; doi:10.1038/sc.2014.186; published online 25 November 2014

\section{INTRODUCTION}

Heterotopic ossification ( $\mathrm{HO})$ is a common complication after spinal cord injury (SCI) and was first described in 1863 by Ried. The incidence among patients with SCI ranges from $16 \%$ to $53 \% .{ }^{1}$ It is also known that patients with $\mathrm{HO}$ after SCI develop deep vein thrombosis ${ }^{2,3}$ and are of a persistent hypercoaguable state. ${ }^{4}$ Therefore increased D-Dimere levels are often noticed in patients with traumatic spinal cord disturbances, but it is not certain if all patients with increased D-Dimere levels develop an $\mathrm{HO}$. $\mathrm{HO}$ is mostly diagnosed between 1-6 months after injury with a peak at 2 months. ${ }^{5,6}$ The role of ultrasound in early detection of HO is well established in clinical practice. ${ }^{7}$ Like in the Brooker classification there are four levels described in the ultrasound classification (lowered echo and dorsal echo increase (I); echo poor tissue (II), echo poor tissue with single bone particles (III) and confluent bone particles (IV)).
The question was if there are high risk patients for the development of $\mathrm{HO}$, regarding age and accompanying injuries or neurologic level and grade. We also wanted to know if there are any standard blood markers to detect $\mathrm{HO}$ at an early stage and if any clinical symptoms can give predictive answer for the occurrence of heterotopic ossification at an early stage in SCI patients. Furthermore, the outcome of the patients after radiation therapy was assessed.

The etiology and risk factors or predictive markers for developing $\mathrm{HO}$ after acute SCI are still not exactly known. Therefore, the main question of this study was to find indicators that are easy to assess and can help to sort out patients with a high risk of HO. Our hypothesis was that younger patients with acute SCIs more often develop HO.

\section{MATERIALS AND METHODS}

This retrospective study included all patients from 2008 to 2012 with acute injuries of the spinal cord treated at the Department of Spinal cord injury, BGTrauma Center Murnau, Germany. Patients suffering from SCI caused non- 
traumatically (e.g., tumor, bleeding due to anticoagulant treatment) were excluded. Age, gender and neurological level of lesion, as well as the AIS score were evaluated. They were divided into a tetra- and paraplegic group. Every 2 weeks a standardized sonography of the hips was performed according to the protocol of Maier. ${ }^{7}$

All patients with suspicious ultrasound results gained a MRI of the pelvis using a 1.5 Tesla MRI gadget. (MR-Scanner Achieva 1.5T (Philips Medical Systems Nederland, 5684 PC Best, The Netherlands).

The sub group of SCI patients suffering of $\mathrm{HO}$ of the hips were extracted and the incidence of $\mathrm{HO}$ was calculated. ${ }^{8}$

Symptoms, concerning swelling of the lower extremity, pain and reduction of hip movement and accompanying injuries of the brain and lung were recorded. We also evaluated D-Dimere levels in acute spinal injury routinely once a week.

For prophylaxis of deep vein thrombosis and $\mathrm{HO}$ all Patients were given low molecular weight heparine and stockings. Patients with $\mathrm{HO}$ underwent a lowfield radiation therapy with five cycles in a row. Afterwards patients were controlled by ultrasound every 2 weeks. Indometacine was not administered.

As statistical method a Fisher's exact test was used for calculation, and a significant correlation was performed using SPSS 19 program (Ehningen, Germany).

\section{RESULTS}

A total of 575 patients with acute spinal injury were re-evaluated retrospectively. Thirty-two of these patients developed HO, which corresponds with an incidence of 5.5\%. The mean age was 42.6 years (range: 17-79). The age disribution amongst the SCI patients without HO was 48 years (range: 14-90). There was no significant correlation between age and development of $\mathrm{HO}(P=0.058$; Figure 1).

The gender distribution of male/female was 30:2, while in patients without $\mathrm{HO}$ a ratio of male/female of 409:138 was observed.

In 12 cases we found tetraplegia and in 20 cases paraplegia. AIS A occurred in 20 cases (64\%), AIS B in $6(19 \%)$ and AIS C and D in 3 cases $(C=8.5 \%, D=8.5 \%)$ each. The relationship between AIS grade and $\mathrm{HO}$ shows a significant correlation $(P=0.048)$. In the normal population of SCI patients AIS A occurred in 223 cases (38\%), AIS B in 77 cases (13\%), C in 141 (25\%) and D in 138 cases (24\%; Figure 2).

Brooker 0 stage was detected in 26 patients, Brooker 1 in two patients, and a Brooker stage of 2 and more in five patients. The adductor muscles were affected in 19 cases, the quadriceps muscle in 15 and the obturatoric muscle in 7 cases. The psoas muscle showed

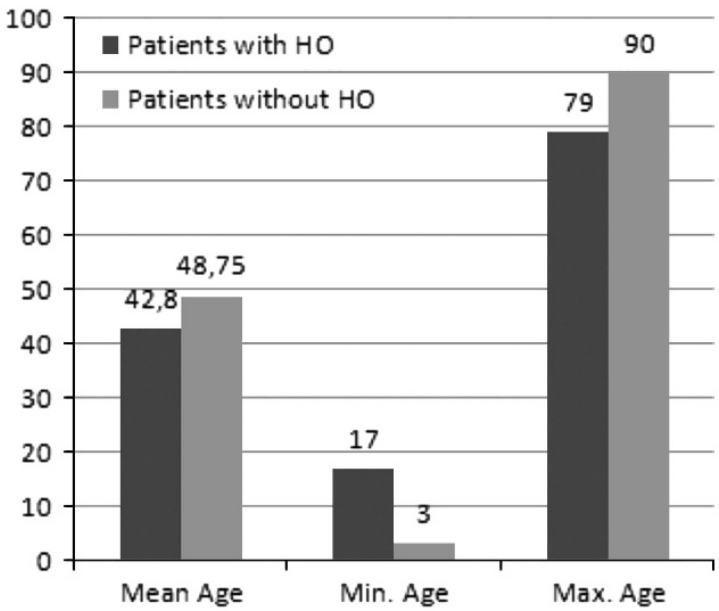

Figure 1 No significant differences regarding age between patients with or without $\mathrm{HO}$ were found. HO, heterotopic ossification; Max., maximum; Min., minimum.
$\mathrm{HO}$ in five cases. Both sides were affected in 22 cases and in 10 cases HO occurred on one side (Figure 3).

In mean $\mathrm{HO}$ occurred after 9 weeks $($ s.d. $=4.6)$ after SCI, with two peaks. One peak was observed after 5 weeks and the other was detected at 9 weeks after SCI. No HO was found after the 22nd week.

An increase of D-Dimere level was observed in 24 cases in mean of $4308 \mathrm{ng} \mathrm{ml}^{-1}$ (range: 1210-14420 $\mathrm{ng} \mathrm{ml}^{-1}$ ). After a duration of 4 weeks after radiation therapy, increased D-Dimere levels were still detectable, but reduced to $50 \%$ compared with the acute phase of $\mathrm{HO}$.

Local symptoms such as swelling and decrease in range of motion (ROM) occurred in 15 cases. Additionally unspecific fever and lassitude was detected in 27 cases. The incidence of thrombosis (four cases) or lung emboli (two cases) was not increased in the population of patients with $\mathrm{HO}$. Accompanying brain injury occurred in 10 cases and thoracic trauma respectively early ventral thoracoscopic spondylodesis occurred in 16 cases.

No patient obtained a further progression of $\mathrm{HO}$ after low-field radiation therapy. After 4 weeks the physiological ripple sign of muscles in ultrasound examination was observed and D-Dimere levels decreased significantly $(P=0.043)$. In patients with Brooker stage $0-\mathrm{I}$ no loss of passive motion was observed. Swelling and redness of the hips was reduced immediately after radiation therapy.

\section{DISCUSSION}

The results of the present study showed an incidence in our SCI population of $5 \%$ and no relationship between age and development of $\mathrm{HO}$ in SCI patients. Owing to the clinical review of van $\mathrm{Kujik}^{9}$ the incidence of $\mathrm{HO}$ in SCI patients ranges between 10-53\% depending on the method of detection. This extreme difference might result because many publications he reviewed were published in an era before ultrasound was established in clinical practice. ${ }^{10-21}$ In later studies the risk for $\mathrm{HO}$ was found to be comparable to ours. ${ }^{22,23}$ One problem in describing $\mathrm{HO}$ might be, that examiners quote $\mathrm{HO}$ in the gluteal region which do not result from neurogenic edema, but from mostly bedding in supine position and permanent wheel-chair dependency which causes high pressure at the gluteal region.

The risk for male SCI patients to develop $\mathrm{HO}$ was five times higher than for female patients. The ratio of male and female SCI patients in our department is 3:1 while the ratio in the population of SCI patients with HO is $15: 1$. Therefore there is a significantly higher risk to develop a HO for male SCI patients than for female. This is contrary to the findings of Knudsen ${ }^{24}$ who did not find any relationship regarding gender. A reason for these different conclusions could not be detected.

When an AIS A was observed there was a higher risk to develop HO compared to patients with AIS D score. Catz et al. ${ }^{25}$ did not find any relationship between grade of paralysis and the development of $\mathrm{HO}$ while in other studies a strong relationship was found. ${ }^{25,26}$ The different findings of Catz et al. ${ }^{25}$ might be caused by the small number of patients providing no power for significance.

Two-third of the cases showed a HO in the medio-ventral muscles of both hips which can be explained by the pathophysiological mechanisms of prolonged immobilization, autonomic dysregulation and neuro-immunological and neuro-humoral changes. By the ongoing demineralization of bone, prostaglandin release and altered $\mathrm{Ca}-\mathrm{P}$ balance there are systemic contact points along the pathway for bone development. ${ }^{27,28}$ The remaining cases of single sided $\mathrm{HO}$ at the hip might be caused by micro trauma during the remobilization process in the physiotherapy, but this is a hypothetic assumption. 
We found a peak in $\mathrm{HO}$ occurrence at the 9th week after injury which is also confirmed by several authors, ${ }^{29-31}$ but pathophysiological explanations could not be found at the moment.

Symptoms such as swelling, decrease of motion of the hips or fever in absence of infection of the lung or urinary tract were found in an early stage of HO. By additional ultrasound and laboratory examination with concern on D-Dimere levels, HO was detected in $98 \%$ of all cases. The D-Dimere levels were increased significantly in 24 cases, but this marker has to be described as too unspecific because increases of D-Dimere are observed in any infection or trauma. In recent studies $^{32-34}$ urinary and serum calcium as well as alkaline phosphatase are described as markers for diagnosis and monitoring of $\mathrm{HO}$, but D-Dimere levels were not described since then. While the neuro-

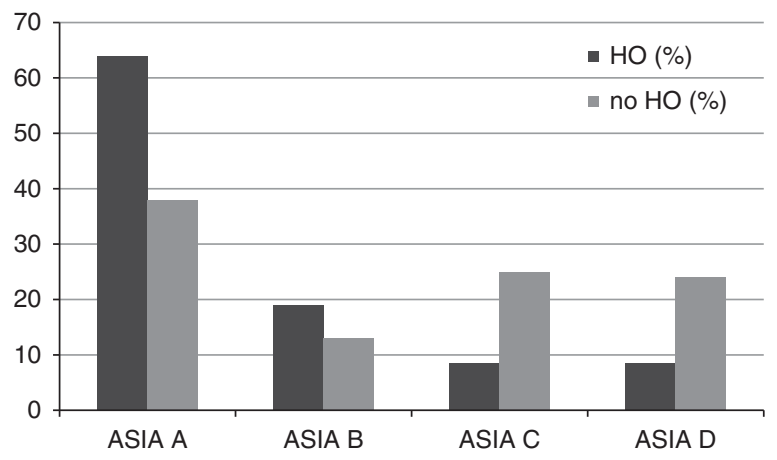

Figure 2 Distribution of patients with and without heterotopic ossification (HO) in correlation of the ASIA Impairment Scale (AIS) grade. humoral system is altered after SCI it can be explained why D-Dimere levels increase during the development of HO. Interestingly, D-Dimere levels decreased after subsequent low - field irradiation during a period of 4 weeks to nearly normal values. During this period ultrasound examination obtained a good monitoring method and a recurrence to normal ripple sign of the muscles occurred. Indometacine was not administered because there is low evidence for a positive effect in neurogenic heterotopic ossifications. ${ }^{35,36}$ In these studies only a small population was examined with no power for statistical significance.

In $50 \%$ of the cases with $\mathrm{HO}$ a concomitant lung injury or thoracoscopic ventral spondylodesis was performed. From animal models it is known, that there is a relation between up-regulation of the BMP/Smad activity by the death of submucosa muscle cells of the lung, ${ }^{37}$ but it was not possible to clarify why patients with SCI develop $\mathrm{HO}$ after thoracoscopic spondylodesis and lung trauma, while patients without SCI, suffering of a thoracic trauma, by injury or iatrogenic lung manipulation, do not. This item has to be further identified.

As limitations of this study we need to give the lack of D-Dimere values before the occurrence of $\mathrm{HO}$ symptoms. We now see the $\mathrm{D}$-Dimere as a promising marker for detecting $\mathrm{HO}$ at an early stage, but further investigations need to clarify the validity of this. We also have to admit that we have no explanation for the high incidence of thoracic trauma which has to be investigated in future, especially with regard to other markers like BMP and TGF- $\beta$.

\section{CONCLUSION}

This study showed that development of heterotopic ossifications in SCI patients is influenced by several risk factors like lung trauma and

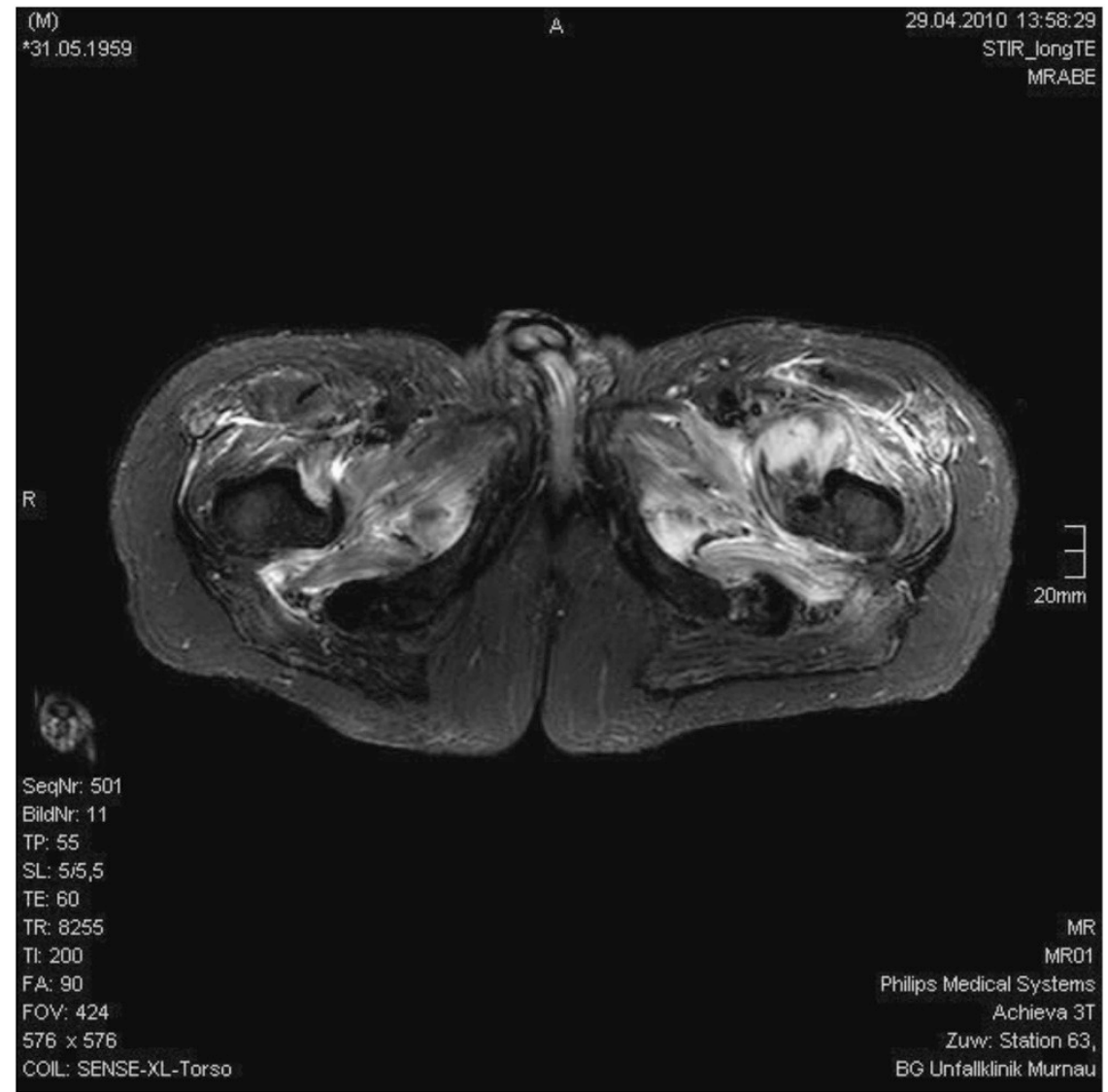

Figure 351 year old patient suffering of HO in the adductor, psoas and quadriceps muscles on both sides, left > right. 
grade of paralysis. Four-digit D-Dimere as a blood marker can be helpful in diagnosing $\mathrm{HO}$, but it is too unspecific to use it as a single diagnostic tool. The outcome after radiation therapy in SCI patients was very good and no loss in passive motion was observed when $\mathrm{HO}$ was detected early. The use of indometacine is unjustifiable.

\section{DATA ARCHIVING}

There were no data to deposit.

\section{CONFLICT OF INTEREST}

The authors declare no conflict of interest.

1 Venier LH, Ditunno JF. Heterotopic ossification in paraplegia patients. Arch Phys Med Rehabil 1971; 52: 475-479.

2 Haselkorn JK, Britell CW, Cardenas DD. Diagnostic imaging of heterotopic ossification with coexisting deep vein thrombosis in flaccid paraplegia. Arch Phys Med Rehabil 1991; 72: 227-229.

3 Colachis SC, Clinchot DM. The association between deep vein thrombosis and heterotopic ossification in patients with acute traumatic spinal cord injury. Paraplegia 1993. 31: 507-512.

4 Perkash A, Sullivan G, Toth L, Bradleigh LH, Linder SH, Perkash I et al. Persistent hypercoagulation associated with heterotopic ossification in patients with spinal cord injury long after injury has occurred. Paraplegia 1993; 31: 653-659.

5 Garland DE. Clinical observations on fracture and heterotopic ossification in the spinal cord and brain injured populations. Clin Orthop 1988; 233: 86-101.

6 Wharton GW, Morgan TH. Ankylosis in the paralyzed patient. J Bone Joint Surg 1970; 52A: 105-112.

7 Maier D. Heterotopic ossification spinal cord injury. Management through early diagnosis and therapy. Orthopade 2005; 34: 120-127.

8 Mahay PR, Urist MR. Experimental heterotopic bone formation induced by bone morphogenetic protein and recombinant human interleukin-1b. Clin Orthop 1988; 237: 236-244.

9 van Kuijk AA, Geurts ACH, van Kuppevelt HJM. Neurogenic heterotopic ossifcation in spinal cord injury. Spinal Cord 2002; 40: 313-326.

10 Heilbrun N, Kuhn WG. Erosive bone lesions and soft tissue ossifcations associated with spinal cord injuries. (Paraplegia) Radiology 1947; 48: 579-593.

11 Abramason AS. Bone disturbances in injuries to the spinal cord and cauda equina. Their prevention by ambulation. J Bone Joint Surg 1948; 30: 982-988.

12 Liberson M. Soft tissue calcifcations in cord lesions. JAMA 1953; 11: 1010-1013.

13 Lodge T. Bone, joint and soft tissue changes following paraplegia. Acta Radiol 1956; 46: 435-445.

14 Damanski M. Heterotopic ossifcation in paraplegia: a clinical study. J Bone Joint Surg 1961; 43: 286-299.

15 Hardy AG, Dickson JW. Pathological ossifcation in traumatic paraplegia. J Bone Joint Surg 1963; 45: 76-87.
16 Freehafer AA, Yurick R, Mast WA. Para-articular ossifcation in spinal cord injury. Med Serv J Can 1966; 22: 471-478.

17 Silver JR. Heterotropic ossifcation. A clinical study of its possible relationship to trauma. Paraplegia 1969; 7: 220-230.

18 Wharton GW, Morgan TH. Ankylosis in the paralyzed patient. J Bone Joint Surg 1970, 52A: 105-112.

19 Hassard GH. Heterotropic bone formation about the hip and unilateral decubitus ulcers in spinal cord injury. Arch Phys Med Rehabil 1975; 56: 355-358.

20 Stover SL, Hataway CJ, Zeiger HE. Heterotopic ossifcation in spinal cord injured patients. Arch Phys Med Rehabil 1975; 56: 199-204.

21 Finerman GAM, Stover SL. Heterotopic ossifcation following hip replacement or spinal cord injury. Two clinical studies with EHDP. Metabl Bone Dis Rel Res 1981; 4/5 337-342.

22 Taly AB, Nair KP, Kumar MV, Jayakumar PN, Vasudev MK, Ravishankar D et al. Heterotopic ossifcation in non-traumatic myelopathies. Spinal Cord 1999; 37: 47-49.

23 Knudsen L, Lundberg D, Ericsson G. Myositis ossifcans circumscripta in para-/ tetraplegics. Scand J Rheum 1982; 11: 27-31.

24 Bravo-Payno P, Esclarin A, Arzoz T, Arroyo O, Labarta C. Incidence and risk factors in the appearance of heterotopic ossification in spinal cord injury. Paraplegia 1992; 30: 740-745.

25 Wittenberg RH, Peschke U, Bötel U. Heterotopic ossifcation after spinal cord injury; epidemiology and risk factors. J Bone Joint Surg 1992; 74: 215-218.

26 Catz A, Snir D, Groswasser Z, Mendelson L, Solzi P. Is the appearance of peri-articular new bone formation related to local neurological disability? Paraplegia 1992; 30: 361-365.

27 Sawyer JR, Myers MA, Rosier RN, Puzas JE. Heterotopic ossifcation. Clinical and cellular aspects. Calcif Tissue Int 1991; 49: 208-215.

28 Lotta S, Scelsi L, Scelsi R. Microvascular changes in the lower extremities of paraplegics with heterotopic ossifcation. Spinal Cord 2001; 39: 595-598.

29 Garland DE, Orwin JF. Resection of heterotopic ossifcation in patients with spinal cord injuries. Clin Orthop 1989; 242: 169-176.

$30 \mathrm{Kim}$ SW, Wu SY, Kim RC. Computerized quantitative radionuclide assessment of heterotopic ossification in spinal cord patients. Paraplegia 1992; 30: 803-807.

31 Citak M, Suero EM, Backhaus M, Aach M, Godry H, Meindl R et al. Risk factors for heterotopic ossifcation in patients with spinal cord injury: a case-control study of 264 patients. Spine (Phila Pa 1976) 2012; 37: 1953-1957.

32 Furman R, Nicholas JJ, Jivoff L. Elevation of the serum alkaline phosphatase coincident with ectopic bone formation in paraplegic patients. J Bone Joint Surg 1970; 52. 1131-1137.

33 Kim SW, Charter RA, Chai CJ, Kim SK, Kim ES. Serum alkaline phosphatase and inorganic phosphorus values in spinal cord injury patients with heterotopic ossifcation. Paraplegia 1990; 28: 441-447.

34 Chantraine A, Nusgens B, Lapiere CM. Biochemical analysis of heterotopic ossifcation in spinal cord injury patients. Paraplegia 1995; 33: 398-401.

35 Banovac K, Williams JM, Patrick LD, Levi A. Prevention of heterotopic ossification after spinal cord injury with COX-2 selective inhibitor (rofecoxib). Spinal Cord 2004; 42: 707-710.

36 Banovac K, Williams JM, Patrick LD, Haniff YM. Prevention of heterotopic ossification after spinal cord injury with indomethacin. Spinal Cord 2001; 39: 370-374.

37 Alexandros Sountoulidis AS, Giaglis S, Apostolou E, Monteiro R, Susana M de Sousa Lopes $\mathrm{C}$ et al. Activation of the canonical bone morphogenetic protein (BMP) pathway during lung morphogenesis and adult lung tissue repair. PLoS One 2012; 7: e41460. 